\title{
Hydrogen on III-V (110) surfaces: Charge accumulation and STM signatures
}

\author{
C. W. M. Castleton, ${ }^{1}$ A. Höglund, ${ }^{2}$ M. Göthelid, ${ }^{3}$ M. C. Qian, ${ }^{2}$ and S. Mirbt ${ }^{2}$ \\ ${ }^{1}$ School of Science and Technology, Nottingham Trent University, Clifton Campus, Nottingham NG11 8NS, United Kingdom \\ ${ }^{2}$ Condensed Matter Theory Group, Department of Physics, Uppsala Universitet, Box 530 S-75121 Uppsala, Sweden \\ ${ }^{3}$ Materialfysik, ICT, KTH, Electrum 229, S-16340 Kista, Sweden
}

(Received 18 February 2013; published 25 July 2013)

\begin{abstract}
The behavior of hydrogen on the 110 surfaces of III-V semiconductors is examined using ab initio density functional theory. It is confirmed that adsorbed hydrogen should lead to a charge accumulation layer in the case of InAs, but shown here that it should not do so for other related III-V semiconductors. It is shown that the hydrogen levels due to surface adsorbed hydrogen behave in a material dependent manner related to the ionicity of the material, and hence do not line up in the universal manner reported by others for hydrogen in the bulk of semiconductors and insulators. This fact, combined with the unusually deep $\Gamma$ point conduction band well of InAs, accounts for the occurrence of an accumulation layer on InAs(110) but not elsewhere. Furthermore, it is shown that adsorbed hydrogen should be extremely hard to distinguish from native defects (particularly vacancies) using scanning tunneling and atomic force microscopy, on both InAs(110) and other III-V (110) surfaces.
\end{abstract}

DOI: 10.1103/PhysRevB.88.045319

PACS number(s): 71.55.Eq, 73.20.Hb, 68.37.Ef, 71.15.Mb

\section{INTRODUCTION}

Considerable effort goes into preparing high quality impurity free semiconductor materials, whether for commercial use or for scientific investigations. The levels of most potential impurities can be reduced to the point of being negligible, but there is one particular impurity that often proves very hard to eradicate: hydrogen. Low levels of hydrogen are almost always present, are hard to detect, and extremely difficult to remove, even under ultrahigh vacuum (UHV) conditions. Indeed it has long been noted that "Hydrogen is present in virtually every step during the processing of III-V devices....". If any growth mechanism could be expected to avoid the presence of hydrogen, it would perhaps be molecular beam epitaxy (MBE), which, at least in principle, only exposes the growth surface to vaporized streams of pure cations and anions. However, hydrogen has, for example, been directly detected on MBE grown InN surfaces. ${ }^{2}$ In this paper we will use density functional theory (DFT) based $a b$ initio methods to examine aspects of the interaction of $\mathrm{H}$ with III-V (110) semiconductor surfaces, discussing charge accumulation and the possible appearance of adsorbed $\mathrm{H}$ in scanning tunneling and atomic force microscopies (STM and AFM).

It has been shown that atomic hydrogen hitting the 110 surfaces of InP and GaAs adheres with a sticking coefficient of 1 (see Ref. 3, and references therein.) The same studies found that desorption occurs around $350{ }^{\circ} \mathrm{C}$ on $\mathrm{GaAs}(110)$, or around $525^{\circ} \mathrm{C}$ on $\operatorname{InP}(110),{ }^{3,4}$ These temperatures do vary with experimental conditions, ${ }^{5}$ but are safely above those used in many surface science investigations. Molecular hydrogen does not dissociate on III-V semiconductor surfaces themselves, ${ }^{6}$ but it does so readily in the presence of a hot filament, such as an ion gauge. In addition, many of the metals and metal oxides used in reaction vessels, vacuum chambers, sample holders, substrates, etc. (including the $\mathrm{Cr}_{2} \mathrm{O}_{3}$ found at the surface of any stainless steel components) can catalyze the dissociation of $\mathrm{H}_{2}$. Atomic $\mathrm{H}$ or $\mathrm{H}^{+}$can enter as a chemical by-product of the feed reagents used to grow the semiconductor materials themselves.
In the case of STM, it is even plausible that dissociation may be caused by the tunnel current itself. Thus any sample placed for investigation inside a vacuum chamber will almost certainly have been exposed to atomic hydrogen. As a result, its presence can be detected on the (110) surfaces of a variety of III-V semiconductors ${ }^{2,5,7}$ using a wide variety of non-imaging based techniques.

The effect of $\mathrm{H}$ on III-V's and other semiconductors has been studied often. ${ }^{7}$ It can stabilize polar (100) surfaces. ${ }^{8}$ It is known to be the cause of supposedly "intrinsic" $n$-type doping in $\mathrm{ZnO}$ (Ref. 9) [where it was found in concentrations of $(6 \pm 2) \times 10^{16} \mathrm{~cm}^{-3}$ in commercially produced "pure" material" $\left.{ }^{10}\right]$, and may be responsible for unintentional doping in InN (Ref. 11) and GaSb. ${ }^{12}$ Furthermore, it has recently been proposed ${ }^{13}$ that adsorbed hydrogen may also be the solution to the long-standing puzzle of charge accumulation on freshly cleaved InAs(110) surfaces.

Charge accumulation - the spontaneous formation of a surface bound two dimensional electron gas (2DEG) - can easily be generated by the presence of impurities at the surface of many different semiconductors, but in the case of (at least) the low index surfaces of InAs it occurs even when cleaving in UHV or after cleaning. ${ }^{14-17}$ Weber et al.${ }^{13}$ recently showed that in most cases this can be accounted for by the presence of In adatoms on the surface, but for freshly cleaved (110) surfaces no such defect should occur, since cleaving only encourages the growth of vacancies, ${ }^{18}$ formed by Langmuir evaporation. ${ }^{19}$ They examined the surface states, and showed that they cannot be used to explain charge accumulation either. Instead, they found that adsorbed atomic hydrogen (hydrogen adatoms, $\mathrm{H}_{\mathrm{ad}}$ ), if present in sufficient amounts, could explain charge accumulation for cleaved $\operatorname{InAs}(110)$, and indeed contribute to it on other InAs surfaces. Surface As ions on InAs(110) have entirely filled dangling bonds, while surface In have empty ones. ${ }^{13,20}$ Weber et al. ${ }^{13}$ showed that hydrogen either attaches as $\mathrm{H}_{\mathrm{ad}}{ }^{+}$to the filled dangling bond on a surface As, with its own electron left over, or attaches as $\mathrm{H}_{\mathrm{ad}}{ }^{-}$to the empty dangling bond on a surface In, with one hole left over. On InAs(110) 
$\mathrm{H}_{\mathrm{ad}}{ }^{+}$has lower energy than $\mathrm{H}_{\mathrm{ad}}{ }^{-}$, so there are always extra electrons which transfer to In dangling bond states, above the conduction band minimum (CBM), and hence lead to a charge accumulation layer. Weber et al. pointed out that since it was due to the way hydrogen interacts with the filled anion and empty cation dangling bonds, this should work on other InAs surfaces too. However, we note below that, by the same argument, this mechanism should also occur on other III-V (110) surfaces, but here we show why that does not occur. We will present and compare results on the absorption structures and plausible hydrogen concentrations for four different III-V's: InAs, InSb, InP, and GaAs (InAs plus the three common semiconductors lying closest in the periodic table). Secondly, we will contrast the occurrence of surface charge accumulation with the universal hydrogen level alignment seen for hydrogen impurities in bulk semiconductors, ${ }^{12}$ and show that alignment is not universal for $\mathrm{H}$ adsorbed on the surface. Finally we will show that hydrogen adsorbed onto III-V (110) surfaces can be very hard to distinguish from intrinsic defects using scanning probe microscopies.

\section{METHOD}

Equilibrium defect concentrations are proportional to $\exp \left(-E_{\mathrm{d}} / k T\right)$, with $E_{\mathrm{d}}$ being the "formation energy" required to create the defect on an otherwise perfect surface:

$$
E_{\mathrm{d}}=E_{\mathrm{T}}\left(\operatorname{def}^{q}\right)-E_{\mathrm{T}}(\text { clean })+\sum_{i} \mu_{i} n_{i}-q\left(\varepsilon_{v}+\varepsilon_{F}\right),
$$

where $E_{\mathrm{T}}\left(\operatorname{def}^{q}\right)$ and $E_{\mathrm{T}}$ (clean) are the total energy of a supercell with and without the charge $q$ defect, which is formed by adding $n_{i}$ atoms of chemical potential $\mu_{i}$. Fermi level $\varepsilon_{F}$ is measured relative to the valence band maximum (VBM) at $\varepsilon_{v}$. We calculate $E_{\mathrm{T}}$ using first principles plane-wave DFT within the local density approximation (LDA), ${ }^{21}$ in a "slab" geometry supercell. Note that, although LDA produces a negative band gap for bulk InAs, the LDA band gap for surface slabs is positive. ${ }^{20}$ We use the VASP code, ${ }^{22}$ with a plane-wave basis (cutoff $240 \mathrm{eV}$ ) and ultrasoft pseudopotenials. Our slab supercell comprises a $(2 \times 4)$ surface supercell with seven atomic layers, separated by a $10 \AA$ vacuum layer. The bottom layer dangling bonds are passivated by pseudo-hydrogens. ${ }^{23}$ The four top layers are allowed to relax until the forces are smaller than $0.01 \mathrm{eV} / \AA$. Brillouin zone integration is on a $2 \times 2 \times 2$ Monkhorst-Pack ${ }^{24}$ grid, with $0.05 \mathrm{eV}$ smearing. The chemical potentials can vary from the cation rich limit (the III-V material in equilibrium with solid In or $\mathrm{Ga}$ ) to the anion rich limit (the III-V in equilibrium with solid P, As, or $\mathrm{Sb}$ ). (Total energies for these reference materials are calculated using the same methods-see Ref. 20 for details.) We plot formation energies over the LDA surface band gap (which is sometimes larger and sometimes smaller than the experimental one), as this gives the most physically consistent $a b$ initio description for the present purposes. The size and impact of this and all other approximations used have been discussed previously, and will not significantly affect the conclusions below $^{20,25}$ most of which depend only on qualitative aspects of the calculated results. (a)

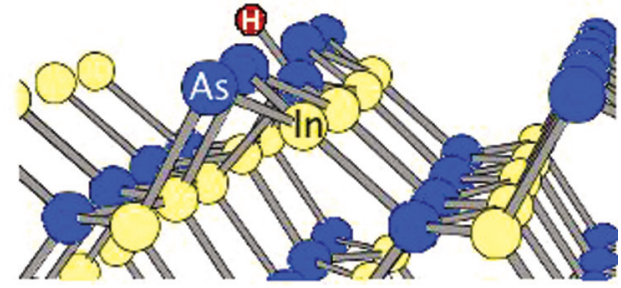

(b)

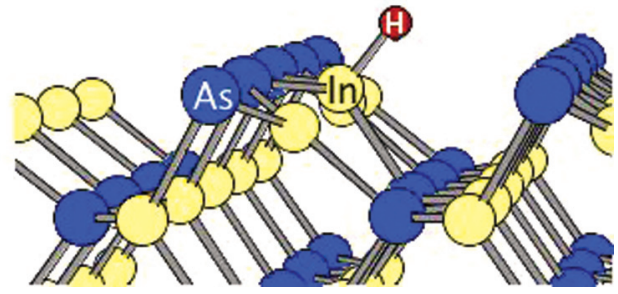

FIG. 1. (Color online) Relaxed geometry of $\mathrm{H}$ adsorbed on InAs at (a) the anion site $\left(\mathrm{H}_{\mathrm{ad}}{ }^{+}\right)$and (b) the cation site $\left(\mathrm{H}_{\mathrm{ad}}{ }^{-}\right)$. Large light (yellow) ions, In. Large dark (blue) ions, As. Small dark (red) ions, H.

\section{H ADSORPTION ON III-V (110) SURFACES}

We consider the adsorption of $\mathrm{H}$ on the (110) surfaces of InAs, InSb, InP, and GaAs. For all four materials, we find two stable adsorption sites: In $p$-type material it is most stable on a surface anion in the +1 charge state $\left(\mathrm{H}_{\mathrm{ad}}{ }^{+}\right)$, while in $n$-type material the most stable site is normally on a surface cation in the -1 charge state $\left(\mathrm{H}_{\mathrm{ad}}{ }^{-}\right)$. This is in agreement with the previous study for $\mathrm{H}$ on InAs (Ref. 13). The neutral and higher charge states are never thermodynamically stable. For some value of $\varepsilon_{F}$ we find a "transfer level" $\varepsilon^{+/-}$at which the stable charge changes directly from +1 to -1 . Similar "negative $U$ " behavior occurs for $\mathrm{H}$ in the bulk for a wide range of semiconductors. ${ }^{12}$ In Fig. 1 we show the relaxed structures for $\mathrm{H}_{\mathrm{ad}}{ }^{+}$and $\mathrm{H}_{\mathrm{ad}}{ }^{-}$on InAs (110) (the other materials are similar), and in Table I we show the relaxed H-surface bond lengths for all four materials. The hydrogenated cation (anion) moves out from (into) the surface, relative to the unoccupied surface ions. As outlined above, $\mathrm{H}_{\mathrm{ad}}{ }^{+}$binds by sharing the two electrons in the filled anion dangling bond, thus stabilizing the surface and forming a normal As- $\mathrm{H}$ bond. Similarly, $\mathrm{H}_{\mathrm{ad}}{ }^{-}$shares its two electrons with the empty cation dangling bond, again stabilizing the surface to form an In-H bond.

Figure 2 shows the formation energies of $\mathrm{H}_{\mathrm{ad}}{ }^{+}$and $\mathrm{H}_{\mathrm{ad}}{ }^{-}$. Following Ref. 27, the chemical potential for $\mathrm{H}$ as a function of temperature $(T)$ and $\mathrm{H}_{2}$ partial pressure $(P)$ is

$$
\mu_{\mathrm{H}}(T, P)=\mu_{\mathrm{H}}\left(0, P^{o}\right)+\mu_{\mathrm{H}}\left(T, P^{o}\right)+\frac{1}{2} k T \ln \left(\frac{P}{P^{o}}\right) .
$$

TABLE I. Bond lengths in $\AA$, between $\mathrm{H}$ and cations/anions on various III-V (110) surfaces.

\begin{tabular}{lcc}
\hline \hline Material & Cation-H & Anion-H \\
\hline InSb & 1.7 & 1.7 \\
InAs & 1.7 & 1.5 \\
InP & 1.7 & 1.4 \\
GaAs & 1.6 & 1.5 \\
\hline \hline
\end{tabular}




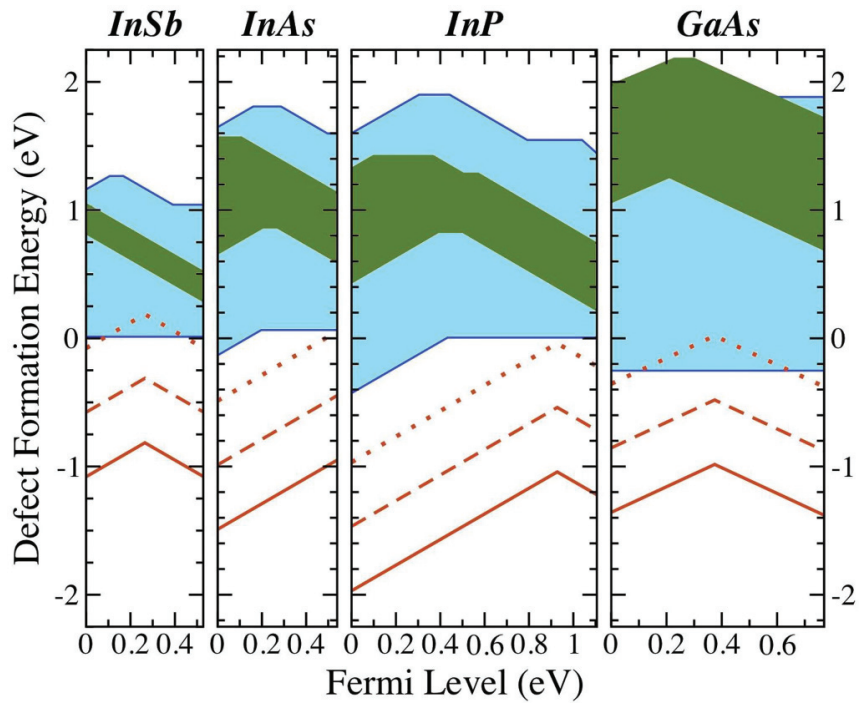

FIG. 2. (Color online) The formation energies of $\mathrm{H}_{\mathrm{ad}}{ }^{+}$and $\mathrm{H}_{\mathrm{ad}}{ }^{-}$ on the (110) surfaces of InSb, InAs, InP, and GaAs (red lines), for $P=$ $10^{-8} \mathrm{~Pa}$ and $T=0 \mathrm{~K}$ (solid), $300 \mathrm{~K}$ (dashed), and $600 \mathrm{~K}$ (dotted). The inner (dark green) shaded area shows vacancy formation energies; the outer (light blue) area shows those of all native defects-data from Ref. 20 except for GaAs, taken from Ref. 29.

The zero-temperature term $\mu_{\mathrm{H}}\left(0, P^{o}\right)$ is taken as $2.25 \mathrm{eV}$ (half the calculated $\mathrm{H}_{2}$ dimer energy, including zero-point energy ${ }^{9}$ ), and lies within $0.01 \mathrm{eV}$ of the experimental value. Its variation with $T$ [second term in Eq. (2)] at standard atmospheric pressure $P^{o}$ is derived from tables. ${ }^{27,28}$ The final term gives the variation with $P$. The $\mathrm{H}_{\mathrm{ad}}{ }^{+}$and $\mathrm{H}_{\mathrm{ad}}{ }^{-}$formation energies are plotted for $T=0,300$, and $600 \mathrm{~K}$, at a typical vacuum chamber partial pressure of $P=10^{-8} \mathrm{~Pa}$.

In order to aid comparison, the ranges of formation energies expected for native defects are also indicated by the shaded regions in Fig. 2 (original data from Refs. 20 and 29). The formation energies of individual defects have a maxima at either the cation or the anion rich stability limit, and a minima at the opposite limit, or vice versa. Here we plot only the lowest and highest native defect formation energies found for each value of $\varepsilon_{F}$ (irrespective of stoichiometry). The darker (green) shaded area indicates the range of formation energies for vacancies alone, since, as mentioned above, while most preparation methods can lead to all native defects, cleaving leads primarily only to vacancies.

For InAs, Fig. 2 confirms the findings of Weber et al.: ${ }^{13}$ the $\varepsilon^{+/-}$level of $\mathrm{H}$ lies within the conduction band, so $\mathrm{H}_{\mathrm{ad}}{ }^{+}$remains more stable than $\mathrm{H}_{\mathrm{ad}}{ }^{-}$even under $n$-type conditions-by $0.1 \mathrm{eV}$ at the LDA surface gap, or by $0.5 \mathrm{eV}$ using the experimental bulk gap. (This compares to $0.3 \mathrm{eV}$ using modified pseudopotentials in the work by Weber $\mathrm{et} \mathrm{al.}{ }^{13}$ ) Therefore, as long as adsorbed hydrogen is the dominant defect, charge neutrality can only be maintained by transferring the electrons from $\mathrm{H}_{\mathrm{ad}}{ }^{+}$to surface CBM states, causing downward band bending and charge accumulation. Furthermore, we have now shown that for reasonable values of $P$ and $T$ adsorbed hydrogen is indeed very likely to be the dominant defect type. On the other hand, Fig. 2 also illustrates why similar charge accumulation does not occur for InSb, InP,

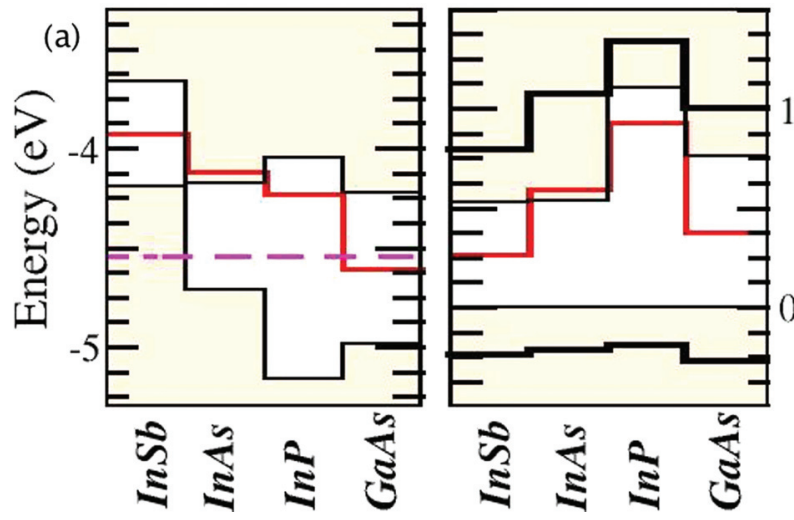

(b)

FIG. 3. (Color online) Valence and conduction bands (shaded) and (surface LDA) band gaps of III-V's, together with $\varepsilon^{+/-}$levels (solid red lines). (a) Levels shown using the band offsets of Ref. 12. The dashed (pink) line shows expected bulk levels according to Ref. 12. (b) Levels shown with no offsets. Bold (black) lines: $k$-point averaged values of the band edges. Details in text.

or GaAs. Adsorbed $\mathrm{H}$ on these materials pins the Fermi level at $\varepsilon^{+/-}$, which lies within even the LDA band gap, with the possible exception of InSb, which is a borderline case possibly warranting future study.

So we confirm that the hydrogen adsorption structures found by Weber et al. on InAs(110) also occur on other III-V's, and that their explanation of accumulation in terms of electron counting for hydrogen attached to surface dangling bonds, resulting in transfer of one electron to the CBM is correct. However, we have also shown that on the other III-V's we have studied it does not lead to charge accumulation because on the other III-V's, $\varepsilon^{+/-}$lies within the gap. So, the real question, which has yet to be addressed, is why does $\varepsilon^{+/-}$lie above the gap for InAs(110), but inside the gap for (at least) the other III-V(110) surfaces considered here?

In Fig. 3(a) we show $\varepsilon^{+/-}$on an "absolute" scale (with respect to the vacuum level), using the band offset based scheme of Van de Walle and Neugebauer. ${ }^{12}$ In bulk materials they showed that $\mathrm{H}$ creates dangling bonds when it interacts with the host lattice, bonding to it in such a manner that $\varepsilon^{+/-}$ has little dependence on the strength of individual $\mathrm{H}$-cation and $\mathrm{H}$-anion bonds. It thus becomes roughly material independent, falling very close to the dashed (pink) line in Fig. 3(a). This "universal line-up" of the bulk $\mathrm{H}$ levels spreads over a wide range of materials, and explains several otherwise puzzling phenomena. ${ }^{12}$ Here, though, we see that at these III-V surfaces the situation is almost the opposite; dangling bonds are already present and $\mathrm{H}$ actually stabilizes (removes) one. We thus find [solid (red) lines in Fig. 3(a)] that the surface values of $\varepsilon^{+/-}$ do not follow the universal line, but are instead rather closely tied to the Brillouin zone averaged conduction band edges (not the CBM value at the $\Gamma$ point itself), as seen in Fig. 3(b). Indeed, $\varepsilon^{+/-}$and the averaged band edge are both similarly proportional to the difference between the host anion and cation covalent radii (related to the degree of ionicity), as shown in Fig. 4, so $\varepsilon^{+/-}$at the surface is specifically material dependent, in contrast to the bulk. In this sense, $\varepsilon^{+/-}$for InAs therefore does nothing unusual at all. Instead, the occurrence of a charge accumulation layer due to $\mathrm{H}$ adsorption on InAs 


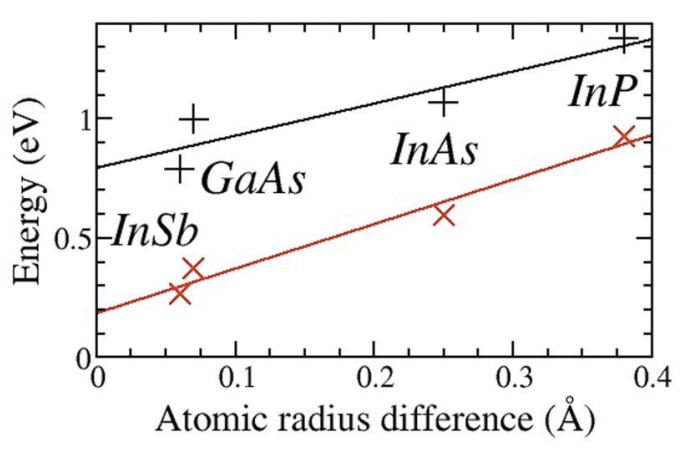

FIG. 4. (Color online) The $k$-point averaged conduction band edge $(+$, black $)$ and $\varepsilon^{+/-}(\times$, red $)$versus the difference in the covalent radii of the host atoms, for InSb, InAs, InP, and GaAs.

but not on InSb, InP, or GaAs is simply due to the singularly deep $\Gamma$ point $C B M$ of InAs, which lies unusually far below the Brillouin zone averaged value of the conduction band edge.

\section{SIMULATED STM FOR H ON III-V (110) SURFACES}

If $\mathrm{H}$ adsorption causes charge accumulation on cleaved InAs(110), and the various other phenomena outlined above, then it should be possible to detect H using STM or AFM. Indeed, Fig. 2 suggests that under many circumstances $\mathrm{H}_{\mathrm{ad}}{ }^{+}$ or $\mathrm{H}_{\mathrm{ad}}{ }^{-}$may even outnumber $V_{\mathrm{As}}{ }^{+1}$ and $V_{\mathrm{In}}{ }^{-1}$ by several orders of magnitude. However to our knowledge it has not so far been identified using imaging experiments on any III-V (110) surface. Indeed for InAs, only $V_{\mathrm{As}}, V_{\mathrm{In}}$, and $\mathrm{S}_{\mathrm{As}}$ dopants have been identified by these methods. ${ }^{30,31}$ We have noted the experimental evidence for the presence of $\mathrm{H}$ on III-V (110) surfaces, and our DFT results also show that it should be stable, but to be visible in STM or AFM it also needs to be at most slow moving on the time scale of the scan. We can find no direct measurements of this in the literature. For bulk III-V's, measurements related to $\mathrm{H}$ mobility vary with experimental method, conditions, doping, etc. For example, in $\mathrm{GaAs}$ the energy barrier has been reported as high as $1.43 \mathrm{eV}$ (Ref. 32) and as low as $0.66 \mathrm{eV}$ (Ref. 33) [in the latter case corresponding to a measure ${ }^{33}$ diffusivity of $\mathrm{O}\left(10^{-12}\right) \mathrm{cm}^{2} / \mathrm{s}$ at
$320 \mathrm{~K}$ ]. Alternatively in $\mathrm{C}$ doped InGaAs thin films it has been reported as $1.9 \mathrm{eV}^{34} \mathrm{As}$ far as surfaces are concerned, there are other surfaces where the STM signatures of $\mathrm{H}$ on the surface are quite distinctive, and so have been seen. For example, on $\mathrm{Ge}(111)-c(2 \times 8),{ }^{35} \mathrm{Si}(111)-p(7 \times 7)$ (Refs. 36 and 37$)$, and $\mathrm{Si}(100)-p(2 \times 1),{ }^{7,38,39}$ As terminated $\operatorname{GaAs}(100)-c(4 \times$ 4) (where it is visible at temperatures up to $400{ }^{\circ} \mathrm{C}$ ) ${ }^{40}$ and in the form of dimers stable at room temperature on $\mathrm{InP}(100)$ and $\mathrm{GaP}(100)$ surfaces. ${ }^{8}$ It was noted that those dimers only moved occasionally at room temperature. ${ }^{8}$ STM videos have been made of adsorbed hydrogen on $\operatorname{Si}(100)-p(2 \times 1)$ becoming mobile around $300-350^{\circ} \mathrm{C}$ and then making individual hops on the order of seconds, ${ }^{41,42}$ corresponding to barrier heights in the $1.0-1.8 \mathrm{eV}$ range. Hence, it is very plausible that $\mathrm{H}$ will move slowly enough to be observable on III-V (110) surfaces, though perhaps not in all cases. (It would be interesting in the future to study the migration barriers of $\mathrm{H}$.)

Turning to the actual appearance of adsorbed $\mathrm{H}$ in scanning probe experiments, for the case of AFM, sensitivity is due to variations in the electrostatic interactions between tip and surface. ${ }^{31}$ The As atoms in AFM on $n$-type InAs(110) therefore appear as bright spots due to the presence of the filled dangling bond protruding from the surface. However, on InAs(110) hydrogen adsorbs on As sites as $\mathrm{H}_{\mathrm{ad}}{ }^{+}$, even in $n$-type material. The As atom then sinks into the surface, and the dangling bond is screened by the $\mathrm{H}$, and so should leave a vacancy-like dark patch in the image.

For STM, Figs. 5, 6, and 7 compare (where available) experimental and simulated images for both adsorbed hydrogen and vacancies on $n$-type $\operatorname{InAs}(110)$ and $p$ - and $n$-type $\operatorname{InP}(110)$. (For InAs only $\mathrm{H}_{\mathrm{ad}}{ }^{+}$and $V_{\mathrm{As}}$ are shown, since $\mathrm{H}_{\mathrm{ad}}{ }^{-}$is never stable.) The simulated images were produced using Tersoff-Hamann theory ${ }^{43}$ applied to the $a b$ initio singleparticle wave functions. Within this formalism, the tunnel current is proportional to the local density of states at the tip position, integrated over the energy range restricted by the applied bias voltage. In general, the simulated STM images of $\mathrm{H}_{\mathrm{ad}}{ }^{+}$turn out to be almost identical to symmetric anion vacancies $\left(V_{\mathrm{A}}\right)$ under both negative (filled state) and to a lesser extent positive (empty state) bias. Meanwhile, those of
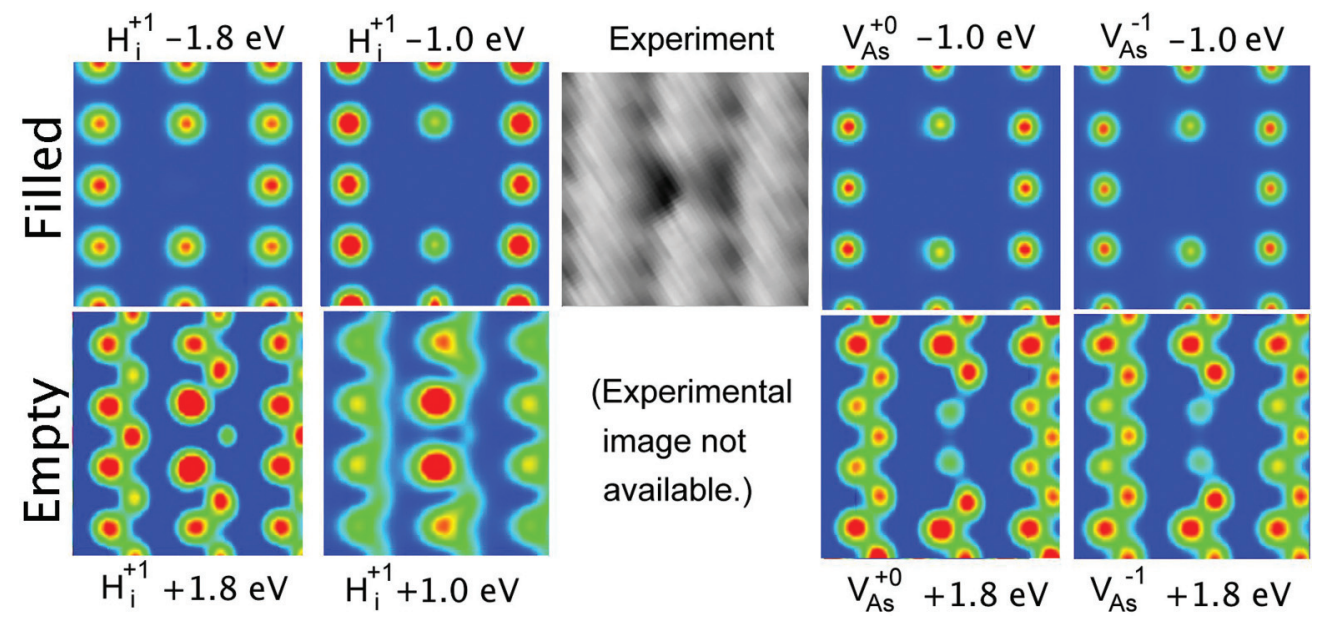

FIG. 5. (Color online) STM images for neutral and negative $V_{\mathrm{A}}$ and $\mathrm{H}_{\mathrm{ad}}{ }^{+}$on InAs(110). Experimental image from Ref. 30 (with kind permission from Springer Science and Business Media). [N.B.: $\mathrm{H}_{\mathrm{ad}}{ }^{-}$is never stable on InAs(110).] The experimental image was obtained at a bias voltage of $-0.9 \mathrm{~V}$, with the tunnel current fixed at $20 \mathrm{pA}$. The area shown is only part of the original $70 \AA \times 70 \AA$ scanned area. 


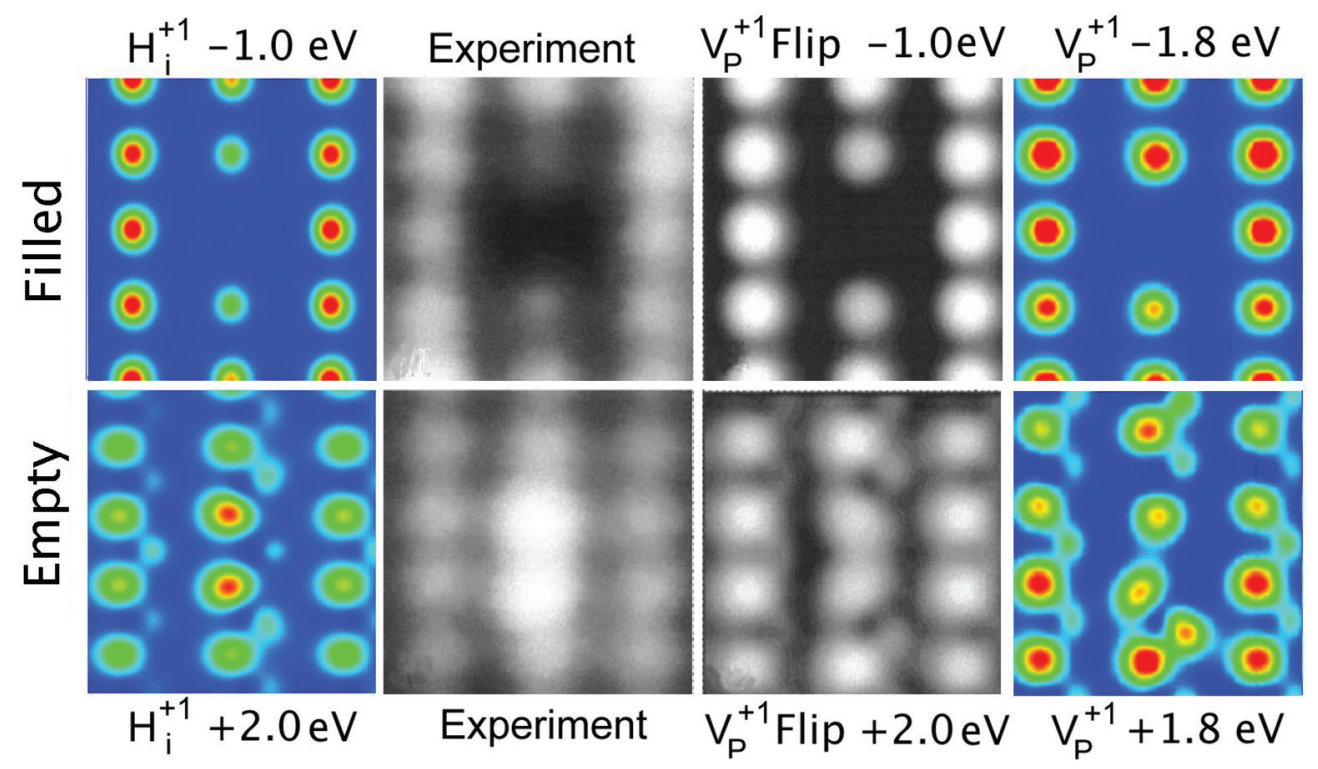

FIG. 6. (Color online) STM images for anion sublattice defects on $\operatorname{InP}(110): \mathrm{H}_{\mathrm{ad}}{ }^{+}$and $V_{\mathrm{P}}$. " $V_{\mathrm{P}}$ flip" refers to the thermal flipping model of Ebert et al.- that and experimental images from Ref. 59.

$\mathrm{H}_{\mathrm{ad}}{ }^{-}$look like symmetric cation vacancies $\left(V_{\mathrm{C}}\right)$, at least under positive bias (empty states). Since this seems to be due to the way $\mathrm{H}$ stabilizes surface dangling bonds it may well apply to other ionic insulators and semiconductors as well. (Indeed, very similar results have recently been obtained for adsorbed hydrogen on cerium dioxide, which looks in STM just like surface oxygen vacancies. ${ }^{44}$ ) Here on the III-V (110) surfaces, the only case that looks significantly different is the (rarely considered experimentally ${ }^{45-58}$ ) case of $\mathrm{H}_{\mathrm{ad}}{ }^{-}$and $V_{\mathrm{C}}$ under negative bias (filled states), where $\mathrm{H}_{\mathrm{ad}}{ }^{-}$does not look like $V_{\mathrm{C}}$, but does look somewhat like a native (or other) adatom, Fig. 7.

Nevertheless, despite the similarities, there are some differences between the simulated images for $\mathrm{H}_{\mathrm{ad}}{ }^{+}$and $V_{\mathrm{A}}$ under positive bias. In particular, the images for $\mathrm{H}_{\mathrm{ad}}{ }^{+}$are symmetric, with a dimming of the signal from the two closest anion neighbors [along the (110) direction], exactly matching the experimental images (Fig. 6, taken from Ref. 59), in contrast to the standard simulated images of $V_{\mathrm{A}}$. The interpretation of these STM images was long controversial, since the experiments ${ }^{45-58}$ show the vacancies as symmetric, but theoretical studies ${ }^{59-64}$ always find them to be Jahn-Teller distorted. The accepted explanation ${ }^{59}$ is that thermal flipping between two degenerate Jahn-Teller distorted structures averages out to the symmetric simulated STM image shown in the center-right in Fig. 6. This is certainly very similar to the experimental image (center left, Fig. 6.) However, unlike adsorbed hydrogen, the thermal flipping model does not reproduce the enhancement of the cations seen in experimental images under positive bias. Nor does it reproduce so clearly the pronounced weakening of the intensity from the neighboring anions seen at negative bias, at least, not without assuming significant and rather anisotropic local band bending. Again, this weakening is very clear in the $\mathrm{H}_{\mathrm{ad}}{ }^{+}$images. These differences are not conclusive, and the experimental images themselves do vary somewhat, ${ }^{45-58}$ with others looking more like the simulated vacancy images, but it does seem likely that at least some of the reported images are due to $\mathrm{H}$ adsorption, not anion evaporation.

There are other contradictions between experimental and theoretical results which may be overcome by assuming the involvement of adsorbed hydrogen. For example, the STM observed concentration of $V_{\mathrm{P}}$ on $p$-type $\mathrm{InP}(110)$ increases with temperature to $435 \mathrm{~K}$, then decreases slightly, possibly to a plateau. ${ }^{19}$ It is believed that this is due to surface creation of $V_{\mathrm{P}}$ increasing with temperature, up to the temperature at which $V_{\mathrm{P}}$ become mobile, after which they diffuse into the bulk, compensating $\mathrm{Zn}$ acceptors. ${ }^{56}$ However, recent theoretical work ${ }^{20}$ showed that, once mobile, $V_{\mathrm{P}}$ should most likely diffuse from bulk to surface, not vice versa. Hence even if the concentration of $V_{\mathrm{P}}$ in the bulk increases with temperature (say, by $V_{\mathrm{P}}-\mathrm{P}_{\mathrm{i}}$ pair creation) the surface concentration should increase faster, not decrease. The decrease can easily be explained if we in fact have a mix of vacancies and adsorbed hydrogen, with the latter starting to disappear as the $\mathrm{H}$ desorption temperature is approached. The acceptor compensation must then have another explanation, but $\mathrm{P}_{\mathrm{In}}, \mathrm{P}_{\mathrm{i}}$, and $\mathrm{In}_{\mathrm{i}}$ are all positively charged $^{20}$ and all have lower bulk formation energies than $V_{\mathrm{P}}$, so there are other possibilities, and combinations are perfectly likely. (Indeed, while the absence of genuine vacancies is very unlikely, one could probably account for the peak in apparent vacancies in STM solely with $\mathrm{H}_{\mathrm{ad}}{ }^{+}$, since dissociation of $\mathrm{H}_{2}$, and hence availability of atomic $\mathrm{H}$ for adsorption, is also a thermally activated process.)

Likewise, STM studies of defect concentrations as a function of time ${ }^{58}$ can also be accounted for by either pure $\mathrm{H}_{\mathrm{ad}}{ }^{+}$or a mix of $V_{\mathrm{A}}$ and $\mathrm{H}_{\mathrm{ad}}{ }^{+}$. The density of "vacancies" in filled-states STM of freshly cleaved $p$-type $\operatorname{InP}(110)$ increases with time, but at an ever-decreasing rate. For $V_{\mathrm{P}}$, the increase would be due to Langmuir evaporation, and the decrease in the rate with time due to the creation of charged vacancies shifting the Fermi level, and the Fermi level shift in turn increasing the vacancy formation energy. For $\mathrm{H}_{\mathrm{ad}}{ }^{+}$, adsorbates would naturally build up with time, and the rate would again naturally 


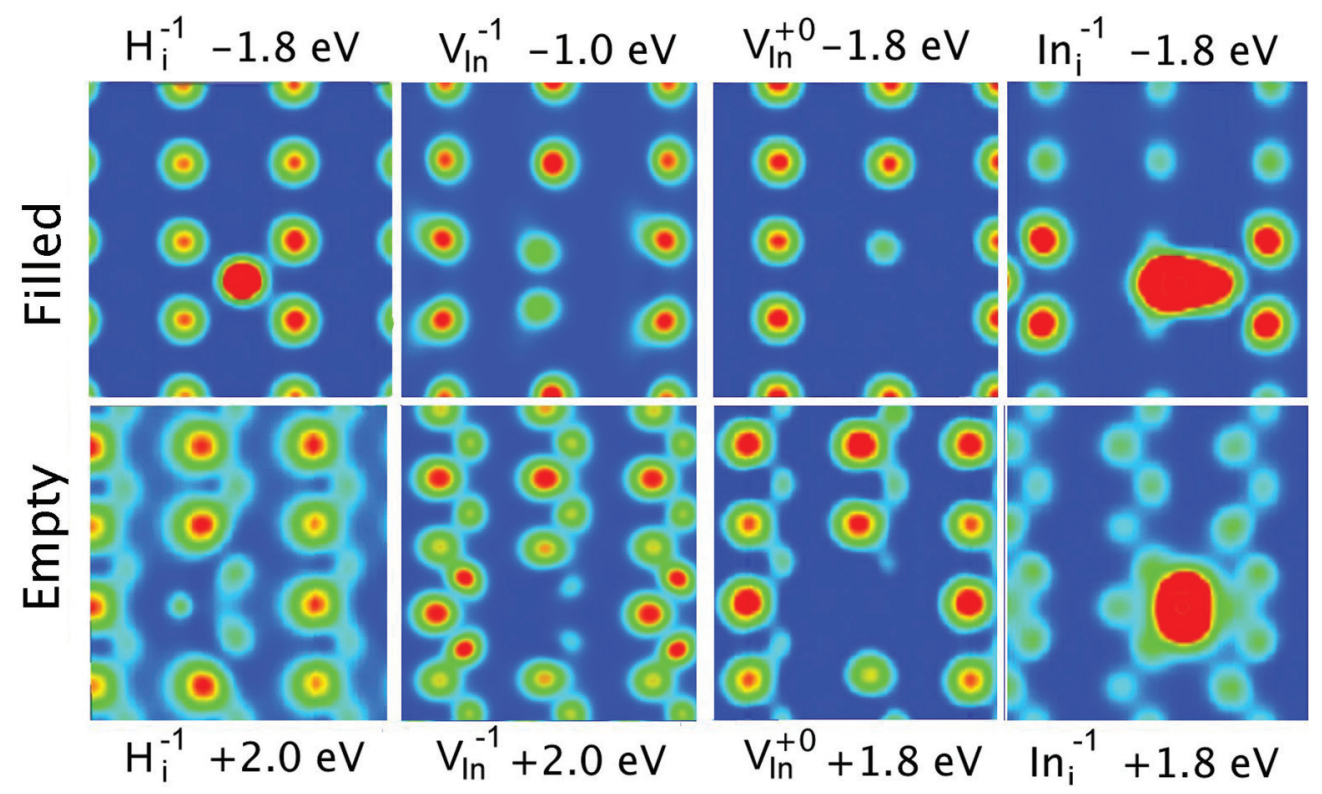

FIG. 7. (Color online) STM images for cation sublattice defects on $\operatorname{InP}(110): \mathrm{H}_{\mathrm{ad}}{ }^{-}$and $V_{\mathrm{In}}$.

decrease due to Fermi level shifts and also a decreased sticking coefficient.

\section{CONCLUSIONS}

We have examined the behavior of adsorbed hydrogen on the (110) surfaces of a selection of III-V materials. We have confirmed the proposal of Weber et al. ${ }^{13}$ that hydrogen adsorption could be the cause of charge accumulation on clean, cleaved InAs surfaces, and we have shown that the same mechanism does not lead to a charge accumulation layer on the (110) surfaces of InSb, InP, or GaAs. Furthermore, we have shown that the $\varepsilon^{+/-}$level of surface adsorbed hydrogen does not follow the "universal line-up" seen for bulk materials, ${ }^{12}$ but instead follows the Brillouin-zone averaged value of the conduction band edge at the surface. This in turn is linked to the difference between the anion and cation covalent radii, which is related to the ionicity, and hence is material dependent. The occurrence of charge accumulation on InAs but not on the other materials is therefore due to the particularly deep $\Gamma$ point valley in the conduction band edge of InAs.

Our calculated formation energies (Fig. 2) suggest that under many conditions $\mathrm{H}$ adatoms may even outnumber surface vacancies. However it is worth emphasizing that we are not suggesting that this will occur under all conditions, or that all of the semiconductor properties currently attributed to surface vacancies are actually due to hydrogen. Vacancies certainly are present (and probably dominant) in many, or most, experiments. For example, the vacancies and vacancy clusters imaged after ion bombardment ${ }^{65}$ or laser ablation ${ }^{57}$ are undoubtedly exactly that. However, our results indicate that significant amounts of adsorbed $\mathrm{H}$ probably are present too in many cases.

Finally, we have shown that AFM and STM images of adsorbed hydrogen on the (110) surfaces of III-V materials are very hard to tell apart from native surface defects: $V_{\mathrm{A}}$ in the case of $\mathrm{H}_{\mathrm{ad}}{ }^{+}, V_{\mathrm{C}}$ in the case of empty state images of $\mathrm{H}_{\mathrm{ad}}{ }^{-}$, or an adatom for filled state images of $\mathrm{H}_{\mathrm{ad}}{ }^{-}$. In the case of $V_{\mathrm{A}}$ and $\mathrm{H}_{\mathrm{ad}}{ }^{+}$, if anything, $\mathrm{H}_{\mathrm{ad}}{ }^{+}$matches the experiments better than the generally accepted thermal flipping model. This may well be a rather general property of hydrogen on at least nonpolar III-V semiconductor surfaces, but on other materials also, as evidenced by the very similar recent results for adsorbed hydrogen on cerium dioxide. ${ }^{44}$ As a result, an STM image apparently showing a dark patch on, say, an anion sublattice, may not always be showing an actual anion vacancy at all. Indeed, this is further complicated on the III-V (110) surfaces because cation vacancies on these surfaces tend to split into anion vacancy/anion-antisite complexes, which again look more like $V_{\mathrm{A}}$ than $V_{\mathrm{C}} \cdot{ }^{66,67}$ In view of all this some literature results, particularly from STM and AFM, may need to be reexamined.

\section{ACKNOWLEDGMENTS}

We thank the Swedish Scientific Council (VR), and the Knut and Alice Wallenberg and the Göran Gustafsson foundations for financial support.
${ }^{1}$ F. Ren, Mater. Sci. Forum 148-149, 141 (1994).

${ }^{2}$ C. S. Gallinat, G. Koblmüller, J. S. Brown, S. Bernardis, J. S. Speck, G. D. Chern, E. D. Readinger, H. Shen, and Michael Wraback, Appl. Phys. Lett. 89, 032109 (2006).

${ }^{3}$ F. Proix, Physica B 170, 457 (1991).
${ }^{4}$ F. Proix, O. Mhamedi, and C. A. Sébenne, J. Vac. Sci. Technol. A 6, 199 (1988).

${ }^{5}$ S. Nannaronea and M. Pedio, Surf. Sci. Rep. 51, 1 (2003).

${ }^{6}$ J. E. Gayone, R. G. Pregliasco, E. A. Sanchez, and O. Grizzi, Phys. Rev. B 56, 4194 (1997). 
${ }^{7}$ A. J. Mayne, D. Riedel, G. Comtet, and G. Dujardin, Prog. Surf. Sci. 81, 1 (2006).

${ }^{8}$ P. Kleinschmidt, H. Döscher, P. Vogt, and T. Hannappel, Phys. Rev. B 83, 155316 (2011).

${ }^{9}$ C. G. Van de Walle, Phys. Rev. Lett. 85, 1012 (2000).

${ }^{10}$ D. M. Hofmann, A. Hofstaetter, F. Leiter, H. Zhou, F. Henecker, B. K. Meyer, S. B. Orlinskii, J. Schmidt, and P. G. Baranov, Phys. Rev. Lett. 88, 045504 (2002).

${ }^{11}$ S. Limpijumnong and C. G. Van de Walle, Phys. Status Solidi B 228, 303 (2001).

${ }^{12}$ C. G. Van de Walle and J. Neugebauer, Nature (London) 423, 626 (2003).

${ }^{13}$ J. R. Weber, A. Janotti, and C. G. Van de Walle, Appl. Phys. Lett. 97, 192106 (2010).

${ }^{14}$ Y. Chen, J. C. Hermanson, and G. J. Lapeyre, Phys. Rev. B 39, 12682 (1989).

${ }^{15}$ M. Noguchi, K. Hirakawa, and T. Ikoma, Phys. Rev. Lett. 66, 2243 (1991).

${ }^{16}$ L.Ö. Olsson, C. B. M. Andersson, M. C. Håkansson, J. Kanski, L. Ilver, and U. O. Karlsson, Phys. Rev. Lett. 76, 3626 (1996).

${ }^{17}$ M. G. Betti, V. Corradini, G. Bertoni, P. Casarini, C. Mariani, and A. Abramo, Phys. Rev. B 63, 155315 (2001).

${ }^{18}$ Some adatoms may form by readsorption of Langmuir evaporated (Ref. 19) ions, but this is generally exothermic (see Ref. 20) so should not contribute significantly.

${ }^{19} \mathrm{Ph}$. Ebert, M. Heinrich, M. Simon, K. Urban, and M. G. Lagally, Phys. Rev. B 51, 9696 (1995).

${ }^{20}$ A. Höglund, C. W. M. Castleton, M. Göthelid, B. Johansson, and S. Mirbt, Phys. Rev. B 74, 075332 (2006).

${ }^{21}$ W. Kohn and L. J. Sham, Phys. Rev. 140, A1133 (1965).

${ }^{22}$ G. Kresse and J. Furthmüller, Comput. Mater. Sci. 6, 15 (1996).

${ }^{23}$ K. Shiraishi, J. Phys. Soc. Jpn. 59, 3455 (1990).

${ }^{24}$ H. J. Monkhorst and J. D. Pack, Phys. Rev. B 13, 5188 (1976).

${ }^{25}$ Finite size scaling to remove supercell size errors (Ref. 26) requires very large supercells indeed for slab geometries. However, in the current case only small changes are noted going to larger (Ref. 20) or smaller (Ref. 13) supercells. Also, since the $\mathrm{H}_{\mathrm{ad}}$ charge is only \pm 1 , the electrostatic errors can be expected to be relatively small, while the ease of relaxation perpendicular to the surface allows strain fields to decay faster than in bulk, so that relatively small elastic errors are expected.

${ }^{26}$ C. W. M. Castleton, A. Höglund, and S. Mirbt, Modell. Simul. Mater. Sci. Eng. 17, 084003 (2009).

${ }^{27}$ K. Reuter and M. Scheffler, Phys. Rev. B 65, 035406 (2001).

${ }^{28}$ D. R. Stull and H. Prophet, JANAF Thermochemical Tables, 2nd ed. (National Bureau of Standards, Washington, D.C., 1971).

${ }^{29}$ G. Schwarz, J. Neugebauer, and M. Scheffler, in Proceedings of the 25th International Conference on the Physics of Semiconductors, Osaka, 2000, edited by N. Miura and T. Ando (Springer, Berlin, 2001), p. 1377.

${ }^{30}$ A. Depuydt, N. S. Maslova, V. I. Panov, V. V. Rakov, S. V. Savinov, and C. Van Haesendonck, Appl. Phys. A 66, S171 (1998).

${ }^{31}$ A. Schwarz, W. Allers, U. D. Schwarz, and R. Wiesendanger, Phys. Rev. B 61, 2837 (2000).

${ }^{32}$ J. M. Zavada and R. G. Wilson, Mater. Sci. Forum 148-149, 189 (1994).

${ }^{33}$ N. M. Johnson, C. Herring, and D. Bour, Phys. Rev. B 48, 18308 (1993).
${ }^{34}$ N. Watanabe, S. Yamahata, and T. Kobayashi, J. Cryst. Growth 200, 599 (1999).

${ }^{35}$ T. Klitsner and J. S. Nelson, Phys. Rev. Lett. 67, 3800 (1991).

${ }^{36}$ K. Mortensen, D. M. Chen, P. J. Bedrossian, J. A. Golovchenko, and F. Besenbacher, Phys. Rev. B 43, 1816 (1991).

${ }^{37}$ J. J. Boland, Surf. Sci. 244, 1 (1991).

${ }^{38}$ J. J. Boland, J. Vac. Sci. Technol. A 10, 2458 (1992).

${ }^{39}$ K. Stokbro, U. J. Quaade, R. Lin, C. Thirstrup, and F. Grey, Faraday Discuss. 117, 231 (2000).

${ }^{40}$ A. Khatiri, J. M. Ripalda, T. J. Krzyzewski, and T. S. Jones, Surf. Sci. 549, 143 (2004).

${ }^{41}$ E. Hill, B. Freelon, and E. Ganz, Phys. Rev. B 60, 15896 (1999).

${ }^{42}$ D. R. Bowler, J. H. G. Owen, C. M. Goringe, K. Miki, and G. A. D. Briggs, J. Phys.: Condens. Matter 12, 7655 (2000).

${ }^{43}$ J. Tersoff and D. R. Hamann, Phys. Rev. Lett. 50, 1998 (1983).

${ }^{44}$ J. Kullgren, M. Wolf, K. Hermansson, and C. W. M. Castleton (unpublished).

${ }^{45} \mathrm{Ph}$. Ebert, G. Cox, U. Poppe, and K. Urban, Ultramicroscopy 42, 871 (1992).

${ }^{46} \mathrm{Ph}$. Ebert and K. Urban, Ultramicroscopy 49, 344 (1993).

${ }^{47} \mathrm{Ph}$. Ebert, K. Urban, and M. G. Lagally, Phys. Rev. Lett. 72, 840 (1994).

${ }^{48}$ G. Lengel, R. Wilkins, G. Brown, M. Weimer, J. Gryko, and R. E. Allen, Phys. Rev. Lett. 72, 836 (1994).

${ }^{49} \mathrm{Ph}$. Ebert, X. Chen, M. Heinrich, M. Simon, K. Urban, and M. G. Lagally, Phys. Rev. Lett. 76, 2089 (1996).

${ }^{50}$ K.-J. Chao, A. R. Smith, and C.-K. Shih, Phys. Rev. B 53, 6935 (1996).

${ }^{51}$ S. Aloni, I. Nevo, and G. Haase, Phys. Rev. B 60, R2165 (1999).

${ }^{52}$ U. Semmler, Ph. Ebert, and K. Urban, Appl. Phys. Lett. 77, 61 (2000).

${ }^{53}$ K. Tanimura and J. Kanasaki, J. Phys.: Condens. Matter 18, S1479 (2006).

${ }^{54}$ A. Laubsch, K. Urban, and Ph. Ebert, Phys. Rev. B 80, 245314 (2009).

${ }^{55} \mathrm{P}$. H. Weidlich, R. E. Dunin-Borkowski, and Ph. Ebert, Phys. Rev. B 84, 085210 (2011).

${ }^{56} \mathrm{Ph}$. Ebert, M. Heinrich, M. Simon, C. Domke, K. Urban, C. K. Shih, M. B. Webb, and M. G. Lagally, Phys. Rev. B 53, 4580 (1996).

${ }^{57}$ J. Kanasaki, Physica B 376, 834 (2006).

${ }^{58}$ U. Semmler, M. Simon, Ph. Ebert, and K. Urban, J. Chem. Phys. 114, 445 (2001).

${ }^{59} \mathrm{Ph}$. Ebert, K. Urban, L. Aballe, C. H. Chen, K. Horn, G. Schwarz, J. Neugebauer, and M. Scheffler, Phys. Rev. Lett. 84, 5816 (2000).

${ }^{60}$ S. B. Zhang and A. Zunger, Phys. Rev. Lett. 77, 119 (1996); 79, 3313 (1997).

${ }^{61}$ H. Kim and J. R. Chelikowsky, Phys. Rev. Lett. 77, 1063 (1996); 79, 3315 (1997); Surf. Sci. 409, 435 (1998).

${ }^{62}$ J. Harper, G. Lengel, R. E. Allen, and M. Weimer, Phys. Rev. Lett. 79, 3312 (1997); 79, 3314 (1997); 80, 643 (1998).

${ }^{63}$ G. Schwarz, A. Kley, J. Neugebauer, and M. Scheffler, Phys. Rev. B 58, 1392 (1998).

${ }^{64}$ M. C. Qian, M. Göthelid, B. Johansson, and S. Mirbt, Phys. Rev. B 66, 155326 (2002).

${ }^{65}$ R. J. Pechman, X.-S. Wang, and J. H. Weaver, Phys. Rev. B 51, 10929 (1995).

${ }^{66}$ A. Höglund, S. Mirbt, C. W. M. Castleton, and M. Göthelid, Phys. Rev. B 78, 155318 (2008).

${ }^{67}$ A. Höglund, S. Mirbt, and C. W. M. Castleton (unpublished). 